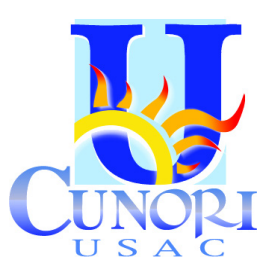

\author{
Revista Ciencia Multidisciplinaria CUNORI \\ http://revistacunori.com \\ DOI: https://doi.org/10.36314/cunori.v2i1.49 \\ ISSN: 2617- 474X (impresa) / 2617- 4758 (en línea)
}

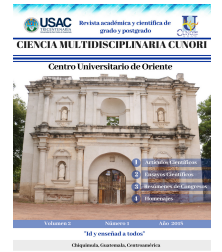

Como citar el artículo

Díaz, R. \& Agustín, N. (2018). La autorregulación académica en los estudiantes de las carreras de ingeniería civil, en ciencias y sistemas e industrial del Centro Universitario de Oriente -Cunori. Revista Ciencia Multidisciplinaria CUNORI, 2(1), 29-34. https:// doi.org/10.36314/cunori.v2i1.49

\title{
La autorregulación académica en los estudiantes de las carreras de ingeniería civil, en ciencias y sistemas e industrial del centro universitario de oriente -CUNORI-
}

\section{Academic self-regulation in the students of civil engineering, science and systems and industrial careers at the university center in the East -CUNORI-}

\author{
Rafael Díaz*, Felipe Agustín \\ Centro Universitario de Oriente -CUNORI, Universidad de San Carlos de Guatemala \\ Recibido: 08 de marzo de 2018 Aceptado: 25 de julio de 2018 \\ Disponible en internet el 17 de agosto de 2018 \\ *Autor para correspondencia. \\ Correo electrónico: rafaeldiaz1902@hotmail.com
}

Resumen

Disten numerosas investigaciones que sostienen que la autorregulación, tiene efecto positivo sobre el rendimiento académico.

$\mathrm{E}$ Kitsantas \& Zimmerman, 2003), encontraron que los estudiantes con calificaciones altas son más estratégicos para planear sus metas, monitorean su propio progreso, autoevalúan su desempeño y conceden un alto valor a los exámenes. La investigación consistió en analizar el proceso de autorregulación académica en los estudiantes de las carreras de Ingeniería Civil, Ingeniería en Ciencias y Sistemas e Ingeniería Industrial del Centro Universitario de Oriente-CUNORI-. El tipo de investigación es descriptiva con enfoque mixto, seleccionándose a 189 estudiantes de ambos sexos, 137 hombres y 52 mujeres del primer, tercer, quinto, séptimo y noveno semestres de las carreras de ingeniería. Se encontró que la autorregulación académica, en los estudiantes de las carreras de ingeniería, están desarrolladas en un nivel medio, generalmente los estudiantes se conforman con ganar los cursos con punteos no muy altos, por lo que hace falta más voluntad y esfuerzo para obtener notas altas. Es también relevante encontrar únicamente el 27 por ciento que en cierta manera corresponde a una población mínima, que si aplica en forma sobresaliente la autorregulación académica, porque tienen el interés, la dedicación y el esfuerzo, la fuerza de voluntad por obtener notas altas en las asignaturas. Por lo que se determina que si existen alumnos autorregulados en las carreras de ingeniería, pero es una la población mínima.

Palabras clave: autorregulación académica, rendimiento académico, conciencia metacognitiva, procesamiento activo, educación superior

\section{Abstract}

$\mathrm{T}$ here are numerous studies that argue that self-regulation has a positive effect on academic performance. (Kitsantas \& Zimmerman, 2003), found that students with high grades are more strategic to plan their goals, monitor their own progress, self-assess their performance and give high value to exams. The research consisted in analyzing the process of academic self-regulation in the students of the Civil Engineering, Engineering in Sciences and Systems and Industrial Engineering careers of the University Center of Oriente -Cunori-. The type of research is descriptive with a mixed approach, selecting 189 students of both sexes, 137 men and 52 women from the first, third, fifth, seventh and ninth semesters of engineering careers. It was found that the academic self-regulation, in the students of the engineering careers, are developed in a medium level, generally the students are satisfied with winning the courses with not very high scores, for what it takes more will and effort to obtain notes high It is also important to find a minimum population that does apply academic self-regulation in an outstanding way, because they have the interest, dedication Los textos publicados en la revista son responsabilidad exclusiva de los autores. 
and effort, the willpower to obtain high marks in the subjects. So we can say that, if there are self-regulated students in engineering careers, but it is a minimum population.

Keywords: academic self-regulation, academic performance, metacognitive awareness, active processing, higher education.

\section{Introducción}

Los profundos cambios que ha experimentado la educación en el nivel superior, han llevado a que el aprendizaje autorregulado se convierta actualmente en un tema central de la investigación y en uno de los ejes primordiales de la práctica educativa. Las características que se les atribuyen a las personas autorreguladoras coinciden con las atribuidas a los estudiantes de alto rendimiento y de alta capacidad, frente a los de bajo rendimiento o que presentan dificultad en el aprendizaje. Existen numerosas investigaciones que ponen en relieve que el uso de estrategias de aprendizaje autorregulado, tienen efectos positivos sobre el rendimiento académico de los estudiantes.

Cuando un estudiante es consciente de la efectividad de las estrategias que utiliza para regular su aprendizaje académico se siente con control y responsable de su propio aprendizaje, se incrementa su motivación para aprender y mejora su rendimiento académico. Lo que claramente los identifica como «autorreguladores» de su aprendizaje no es tanto su utilización aislada de estrategias de aprendizaje, sino su iniciativa personal, su perseverancia en la tarea y las competencias exhibidas, independientemente del contexto en el que ocurre el aprendizaje. Los alumnos «autorreguladores» se centran en su papel como protagonistas de su propio aprendizaje, son conscientes de que el éxito académico depende sobre todo de su actividad e implicación. Idem. De forma más específica la autorregulación implica, el establecimiento de metas y objetivos de aprendizaje, la selección y utilización de estrategias de aprendizaje ajustadas a las demandas de las tareas, el mantenimiento de altos niveles de autoeficacia para aprender y, la regulación, monitorización y evaluación de los propios niveles de ejecución académica.

Por su parte (Winne, 1998) afirma que el alumno debe tener conciencia de sus propios procesos mentales, psicológicos y saber controlar estos procesos, mediante la organización, dirigiendo y ajustándolos a las exigencias de la tarea. Sin embargo para (Kaplan, 2008) la autorregulación no puede concebirse como un constructo unitario en sí mismo como tampoco es un set de estrategias cognitivas, metacognitivas y conductuales, sino que hace referencia al modo en que el estudiante se sitúa frente a la tarea de aprendizaje.

El comportamiento autorregulado refleja su compromiso con la tarea, su deseo de realizarla y, por lo tanto compromete su motivación y su voluntad. Según (Woolfolk, 2010), la metacognición hace referencia a los procesos de pensamiento que la persona tiene acerca de su propio sistema cognitivo (contenidos, procesos, capacidades, limitaciones) y, por otra parte, a los efectos reguladores que tal conocimiento puede ejercer en su actividad; la metacognición entendida como regulación y control de la actividad cognitiva implica la participación activa y responsable del sujeto en los procesos de aprendizaje, y la autorregulación, no es más que el "control ejecutivo" del conocimiento. Por otro lado, la autorregulación es esencialmente un saber procedimental que muchas veces no requiere de ser declarado sino sólo de ser ejecutado al adoptar estrategias de control, también llamadas estrategias o habilidades autorreguladoras que incluyen planificación, monitoreo o supervisión y revisión. 
Para (Pintrich, 2000), el aprendizaje autorregulado se define como un proceso activo y constructivo por el cual el estudiante establece sus propios objetivos de aprendizaje, procurando monitorizar, regular y controlar sus pensamientos, su motivación y su comportamiento de acuerdo a dichos objetivos. Para que el aprendizaje autorregulado se pueda producir, es necesario que la persona ponga en marcha las acciones necesarias para el desarrollo de lo que se conoce como aprendizaje autónomo, auto-determinado o auto-instrucción. Se puede afirmar que el alumno autorregulado es aquél que mejora su rendimiento académico al regular su proceso de aprendizaje, es decir, cuando toma en consideración los conocimientos previos, la zona de desarrollo próximo, el contexto, la motivación, los sentimientos y las emociones.Entre las principales características de los alumnos autorregulados según (Torre, 2007) es ser consciente que la autorregulación está relacionada con el rendimiento académico. Saber cuáles son sus capacidades y conocimientos previos; esto le ayuda a elegir las estrategias que le permitirán un aprendizaje eficiente y eficaz. Conocer sus emociones.

Las negativas las sabe controlar y logra así una mayor motivación. Saber elegir estrategias autorreguladoras cognitivas, metacognitivas, motivacionales y de apoyo. Supervisar la forma en que aprende, revisar constantemente sus hábitos y estrategias de aprendizaje, cambiar si lo estima necesario. Lograr crear ambientes favorables de estudio y aprendizaje, tomar en cuenta el lugar, ubicación, horario, distractores externos e internos. Las estrategias autorreguladoras que aplica al momento de aprender, las transferirá a la vida cotidiana. En la investigación de la autorregulación académica en los estudiantes de las carreras de Ingeniería Civil, Ingeniería en Ciencias y Sistemas e Ingeniería Industrial, se tomó como sustento teórico, el modelo de (Torre, 2007), la cual consiste en cuatro fases para explicar, cómo regulan los estudiantes su aprendizaje en el aula.

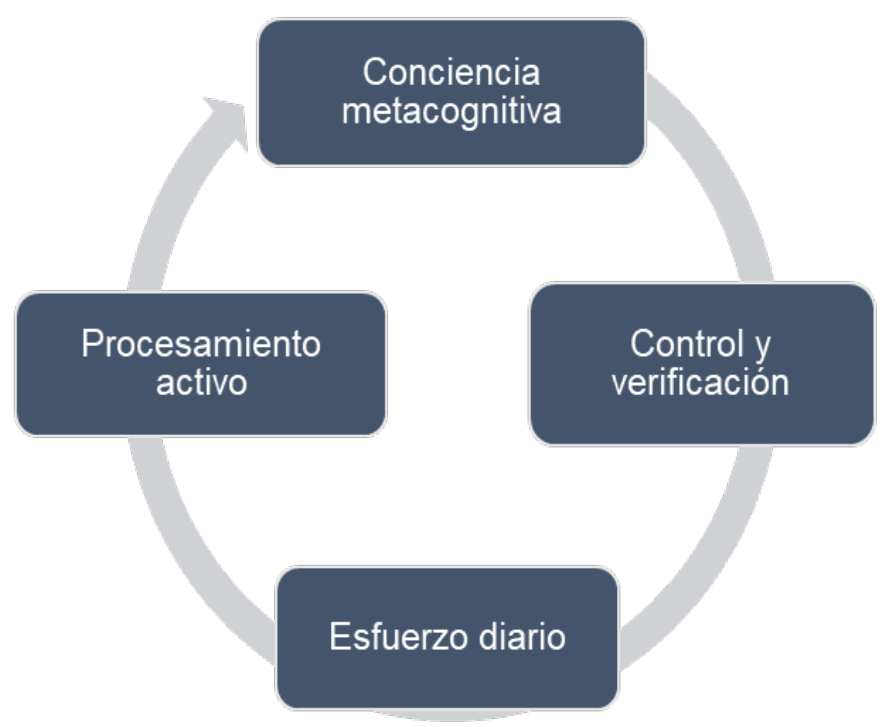

Figura 1: Modelo de Torre, autorregulación académica 
La primera fase es conciencia metacognitiva, es la preparación-planificación y activación, que consiste en determinar los objetivos, activar pre saberes y poner en marcha procesos de planificación conductual y la activación de las percepciones relativas tanto a la tarea, como a la persona y el contexto en que se ubica. En la segunda fase, control y verificación, significa que al regularse y no obtener los resultados esperados debe adaptar o cambiar las estrategias cognitivas y motivacionales que aplica, se analiza la conducta y el contexto. La tercera fase, se refiere al esfuerzo en la realización de las tareas, implica tener en cuenta la conciencia metacognitiva, emocional y conductual sobre la ejecución de la acción de aprendizaje, consiste en controlar efectos, pasos, o cambios que se dan en las diferentes áreas. En la última fase, procesamiento activo, involucra el proceso de evaluación, tanto del docente como del mismo estudiante, es la reflexión sobre la tarea, considera la reacción emocional y la conductual. En virtud de lo anterior, la investigación consistió en analizar el proceso de autorregulación académica en los estudiantes de las carreras de Ingeniería Civil, Ingeniería en Ciencias y Sistemas e Ingeniería Industrial del Centro Universitario de Oriente -Cunori-.

\section{Materiales y métodos}

El tipo de investigación es descriptiva con enfoque mixto, seleccionándose a 189 estudiantes de ambos sexos, 137 hombres y 52 mujeres del primer, tercer, quinto, séptimo y noveno semestres de las carreras de ingeniería. El método que se utilizó es el hipotético-deductivo, que a través de la observación se identificó el problema el cual es el bajo rendimiento académico de los alumnos. El instrumento para recabar datos es por medio de la encuesta con escala Likert, los ítems del cuestionario se basaron en la autorregulación para el aprendizaje académico, validada por Torre, en su tesis doctoral en el año 2007, para la Universidad Pontificia de Comillas en Madrid.

El cuestionario permitió examinar patrones de autorregulación académica en las fases conciencia metacognitiva, control y verificación, esfuerzo diario y procesamiento activo. El cual está conformado por 20 reactivos, que se responden según categorías que van de nunca, casi nunca, algunas veces, casi siempre y siempre. Arrojando una calificación general que oscila entre 20 y 100 puntos. Se tomó en cuenta los datos demográficos como la edad, género, título que posee, tipo de educación privada o mixta, carrera que estudia, semestre que cursa para determinar cuáles son los rasgos que diferencian a los alumnos más motivados frente a los menos motivados.

\section{Resultados}

Los hallazgos se obtuvieron mediante la información que proporcionaron 189 alumnos de las carreras de ingeniería del CUNORI en relación a las siguientes variables: Conciencia metacognitiva, Control y verificación, Esfuerzo en la realización de las tareas y Procesamiento activo. Para tener una mejor comprensión del origen de los resultados, se considera importante presentar el cuadro con las fases de la autorregulación académica y los niveles de autorregulación académica. 


\begin{tabular}{cccccc}
\hline $\begin{array}{c}\text { Niveles de } \\
\text { autorregulación } \\
\text { académica }\end{array}$ & $\begin{array}{c}\text { Fases de la autorregulación académica } \\
\text { Conciencia } \\
\text { metacognitiva }\end{array}$ & $\begin{array}{c}\text { Control y } \\
\text { verificación }\end{array}$ & $\begin{array}{c}\text { Esfuerzo } \\
\text { en las tareas }\end{array}$ & $\begin{array}{c}\text { Total, } \\
\text { Procesamiento } \\
\text { activo }\end{array}$ & $\begin{array}{c}\text { autorregulación } \\
\text { académica }\end{array}$ \\
\hline Muy baja & $0.00 \%$ & $0.00 \%$ & $0.50 \%$ & $1.60 \%$ & $0.00 \%$ \\
Baja & $10.10 \%$ & $4.20 \%$ & $30.20 \%$ & $23.80 \%$ & $4.20 \%$ \\
Media & $68.30 \%$ & $45.50 \%$ & $54.50 \%$ & $55.00 \%$ & $68.80 \%$ \\
Alta & $21.70 \%$ & $50.30 \%$ & $14.80 \%$ & $19.60 \%$ & $27.00 \%$ \\
Total & $100 \%$ & $100 \%$ & $100 \%$ & $100 \%$ & $100 \%$ \\
\hline
\end{tabular}

Cuadro 1. Percepción de la autorregulación académica por cada sub variable.

Los alumnos desarrollan la fase de conciencia metacognitiva en un 68.3 por ciento en un nivel medio e inferior al medio. En la fase de control y verificación los alumnos desarrollan en un 50.3 por ciento en un nivel alto, por mantener una motivación alta, al estudiar están conscientes que con esfuerzo y dedicación se pueden alcanzar los objetivos propuestos. En la fase de esfuerzo en la realización de las tareas los alumnos la desarrollan en un 54.5 por ciento en un nivel medio e inferior al medio. En la última fase la de procesamiento activo, los alumnos, la desarrollan en un 55 por ciento en un nivel medio e inferior al medio.

\section{Discusión}

De acuerdo a los resultados obtenidos en cada sub variable de la autorregulación académica de las carreras de Ingeniería Civil, Ingeniería en Ciencias y Sistema e Ingeniería Industrial, los alumnos algunas veces tienen el compromiso en establecer metas, objetivos y estrategias de aprendizaje para alcanzar altos resultados académicos, lo que no les permite tener una buena ejecución y la posibilidad de verificar los resultados obtenidos comparándolos con lo planeado. Los alumnos estudian casi siempre con el mayor esfuerzo para obtener notas altas.

Únicamente el 27 por ciento que en cierta manera es una población mínima de alumnos los que tienen bien definidos objetivos, metas y estrategias de aprendizaje para alcanzar resultados académicos altos. Los estudiantes que tienen el propósito de conseguir el mejor rendimiento académico posible, tienen control sobre los resultados, se anticipan, se preparan para la tarea, planifican como lo van llevar a cabo, revisan las características de las tareas, controlan en qué medida sus pensamientos, sentimientos y acciones le impulsan a la consecución de metas. También hacen una reflexión sobre sí mismos de lo aprendido a través de una evaluación de objetivos, estrategias y resultados. 


\section{Referencias bibliográficas}

Kaplan, A. (2008). Clarifying Metacognition, Self-Regulation, and Self-Regulated Learning: What's the Purpose? Educational Psychology Review, 20 (4), 477-484.

Kitsantas, A. \& Zimmerman, B. J. (2003). Impact of students' self-efficacy for learning beliefs on their self-regulated learning processes. A paper presented at the Anual convention of the American Educational Research Association, Chicago, IL.

Pintrich, R. (2000). The role of goal orientation in self-regulated learning. En Monique Boekaerts, Paul R. Pintrich, y Moshe Zeidner (eds.), Handbook of self-regulation (pp.452-502). San Diego, CA: Academic Press.

Torre, J. (2007). La autoeficacia, la autorregulación y los enfoques de aprendizaje en estudiantes universitarios. Tesis doctoral, Madrid: Universidad Pontificia Comillas.

Winne, P. (1998). A metacognitive view of individual differences in self-regulated learning. Learning and Individual Differences, 8, 327-353.

Woolfolk, A. (2010). Psicología educativa. (10ª ed.). México: Pearson educación

Zimmerman, B. (2000). El logro de la autorregulación: Una perspectiva cognitiva social. En M. Boekaerts, P.R. Pintrich y M. Zeidner (Eds). Manual de la autorregulación (pp. 451- 502) San Diego, CA: Academic Press.

\section{Sobre el autor}

\section{Rafael Díaz Jacobo}

Licenciado en Administración Educativa y Maestro en Docencia Universitaria con Orientación en Estrategias de Aprendizaje egresado de la Universidad de San Carlos de Guatemala. Investigador del tema "Autorregulación Académica“. Por más de 20 años ha sido profesor de Matemática en Nivel Medio en el Ministerio de Educación y profesor en la Universidad Internaciones.

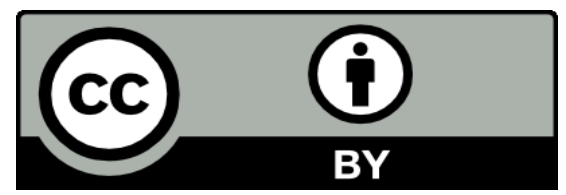

Este texto está protegido por una licencia CreativeCommons 4.0.

Usted es libre para compartir, copiar y redistribuir el material en cualquier medio o formato y adaptar el documento, remezclar, transformar y crear a partir del material para cualquier propósito, incluso comercialmente, siempre que cumpla la condición de atribución: usted debe reconocer el crédito de una obra de manera adecuada, proporcionar un enlace a la licencia, e indicar si se han realizado cambios. Puede hacerlo en cualquier forma razonable, pero no de forma tal que sugiera que tiene el apoyo del licenciante o lo recibe por el uso que hace. 